\title{
Determinazione quantitativa di HIV-|| DNA nel follow-up del paziente HIV-I infetto: esperienza in un gruppo di soggetti HIV-I infetti
}

\section{Francesca Vitone, Davide Gibellini, Pasqua Schiavone, Isabella Bon, Maria Carla Re}

Sezione di Microbiologia, Dipartimento di Medicina Clinica Specialistica e Sperimentale, Università degli Studi di Bologna, Via Massarenti, 9 - 40138 Bologna

Kew words: HIV-I, Real time PCR, HIV-I proviral DNA.

\section{Quantitative detection of HIV-I DNA in the follow-up of HIV infected patients}

\section{SUMMARY}

Proviral HIV-I DNA can represent an alternative viral marker to RNA viral load during the follow-up of HIV-I infected individuals. Sequential blood samples of 12 patients under antiretroviral treatment from 1997 with two NRTIs, who refused to continue any antiviral regimen, were analyzed for 16-20 weeks to study the possible relationship between DNA and RNA viral load.

Results obtained showed an increase or a rebound in viral DNA, quantified by SYBR green real-time PCR in peripheral blood mononuclear cells in most patients, suggesting that the absence of therapy reflects an increase and/or a persistence of cells containing viral DNA. Even though plasma HIV RNA levels remain the basic parameter to monitor the intensity of viral replication, the results obtained seem to indicate that DNA levels could represent an adjunct prognostic marker in monitoring HIV-I infected subjects.

\section{INTRODUZIONE}

Nel corso degli ultimi anni, l'introduzione nella pratica clinica della terapia antiretrovirale ha profondamente modificato la storia naturale dell'infezione da virus dell'immunodeficienza acquisita di tipo 1 (HIV-1), portando ad un miglioramento della qualità di vita dei pazienti oltre che ad una drastica riduzione della mortalità specifica (14).

Nonostante gli importanti successi dei nuovi approcci terapeutici, il virus è comunque in grado di persistere in numerose cellule e tessuti, difficilmente raggiungibili dai farmaci oggi in uso. È evidente che la presenza di questi reservoir, presenti fin dai primi stadi dell'infezione rappresenta uno dei principali ostacoli alla eradicazione dell'infezione giocando un ruolo essenziale nella patogenesi $(1,3,9,10)$. Riuscire, quindi, ad ottenere informazioni relative alla presenza del virus ed al suo outcome diventa di primaria importanza nella gestione del paziente HIV-1 infetto. La quantificazione di DNA provirale, in grado di persistere a dispetto delle terapie in atto nei linfomonociti circolanti, costituisce l'unico parametro quantificabile, permettendo l'apertura di interessanti prospettive. Accanto, quindi, a parametri quali la carica virale plasmatica, il livello dei linfociti $\mathrm{CD} 4+$ e la determinazione delle mutazioni correlate a farmacoresistenze, oggi considerati i gold standard per impostare una corretta terapia farmacologia e seguirne l'efficacia nel tempo, la determinazione della presenza del DNA provirale nei diversi compartimenti cellulari e la sua quantificazione con le eventuali variazioni nel tempo possono rappresentare un utile marker nel followup del paziente infetto $(2,4,11)$. In particolar modo, la possibilità di potere quantificare il DNA in soggetti con valori di RNA plasmatici non evidenziabili potrebbe fornire indicazioni estremamente importanti ai fini prognostici $(5,13,16$, 20).

Anche se recentemente sono stati intrapresi diversi studi per individuare nuove strategie mirate a colpire, sia da un punto di vista terapeutico sia da un punto di vista immunologico (4), il potenziale serbatoio di HIV, non sempre i risultati sembrano essere estremamente confortanti. Se da una parte il calo drastico della viremia plasmatica può portare a giustificati ottimismi, la presenza di virus in grado di albergare in determinati distretti cellulari pone nuovi interrogativi e ci induce ad uno studio più approfondito, sia per cercare di chiarire $i$ tempi di persistenza del virus (integrato e non) a livello nucleare sia per cercare di trovare nuove molecole in grado di colpire questi reservoir virali (18).

Alla luce di tali riflessioni e al fine di chiarire l'eventuale significato prognostico della quantificazione del DNA provirale, come proseguo di studi 
già iniziati dal nostro gruppo di ricerca $(5,6,20)$ abbiamo analizzato per un periodo variabile da 6 a 9 mesi, un piccolo gruppo di pazienti sieropositivi che avevano deciso di interrompere, spontaneamente, la terapia antiretrovirale, nonostante presentassero elevati livelli di carico virale. In particolare, abbiamo valutato, mediante un saggio di PCR real-time, l'andamento di HIV-1 DNA nei linfomonociti ottenuti dal sangue periferico in relazione ai livelli di viremia plasmatici e dei linfociti $\mathrm{CD} 4+$, al fine di ottenere informazioni per una corretta gestione clinica e terapeutica del paziente stesso.

\section{MATERIALI E METODI Pazienti}

Abbiamo studiato, dopo consenso informato, 12 soggetti HIV-1 positivi, sottoposti a terapia antiretrovirale dal 1997 con due analoghi nucleosidici (stavudina e lamivudina, o zidovudina e lamivudina o zidovudina e zalcitabina o zidovudina e didanosina), i quali, nonostante la presenza di livelli elevati di HIV-1 RNA (valore medio: $3.7 \times 10^{3} \mathrm{HIV}-\mathrm{RNA} / \mathrm{ml}$ ), avevano deciso di interrompere volontariamente la terapia.

In tutti i pazienti inclusi nello studio abbiamo esaminato i livelli plasmatici di RNA e quantificato il DNA intracellulare, insieme al numero dei linfociti CD4+ circolanti, dal momento dell'interruzione di terapia (tempo 0) fino a 16-36 settimane, con intervalli mensili.

\section{Quantificazione del carico virale a RNA}

Il sangue di ogni paziente, ottenuto attraverso un prelievo endovenoso, è stato raccolto in provette contenenti EDTA e processato entro tre ore dall'arrivo in laboratorio. Il plasma è stato separato dalla frazione cellulare attraverso centrifugazione a $2500 \mathrm{rpm}$ per 20 minuti e congelato a $-80^{\circ} \mathrm{C}$ prima dell'uso. La quantificazione del carico virale RNA è stata eseguita con il kit commerciale Quantiplex HIV-1 RNA 3.0 Assay (bDNA).

\section{Quantificazione del carico provirale a DNA}

I linfomonociti (PBMC) sono stati separati mediante Ficoll e i pellet, ottenuti dai singoli campioni, contenenti $5 \times 10^{6}$ cellule, sono stati congelati a $-80^{\circ} \mathrm{C}$ fino al loro utilizzo per l'estrazione del DNA. L'estrazione è stata eseguita mediante il kit DNAeasy tissue (Quiagen) e il DNA estratto è stato quantificato, attraverso lettura allo spettrofotometro, alla lunghezza d'onda di $260 / 280 \mathrm{~nm}$. Per l'amplificazione è stata utilizzata una coppia di primer specifici nei confronti di una regione estremamente conservata del gene gag di 142 bp. Il dosaggio del numero di copie di DNA provirale è stato effettuato mediante il saggio di PCR quantitativa real-time, messo a punto nel nostro laboratorio sullo strumento Light-Cycler
(11). In breve, il saggio di SYBR Green Real Time è stato allestito in una soluzione di $20 \mu \mathrm{l}$ finali costituita da $10 \mu 1$ di $2 X$ Quantitect SYBR Green PCR Master Mix (Qiagen), contenente HotStarTaq DNA polimerasi, $0.5 \mu$ di ogni primer alla concentrazione di $25 \mathrm{nM}$ e DNA estratto dai linfociti dei pazienti o dalle diluizioni scalari della curva di riferimento, in quantità di 600 ng. L'amplificazione del gene gag tramite LightCycler è stata svolta nel seguente modo: attivazione della HotStarTaq DNA polimerasi a $95^{\circ} \mathrm{C}$ per 15 minuti; 45 cicli suddivisi in quattro step: $94^{\circ} \mathrm{C}$ per 10 secondi, $60^{\circ} \mathrm{C}$ per 30 secondi, $72^{\circ} \mathrm{C}$ per 30 secondi e $78^{\circ} \mathrm{C}$ per 3 secondi. Alla fine dell'amplificazione è stata eseguita un'analisi della temperatura di Melting del prodotto ottenuto tramite un lento e progressivo aumento della temperatura $\left(0.1^{\circ} \mathrm{C} /\right.$ secondo) fino a $95^{\circ} \mathrm{C}$. Il profilo di amplificazione del bersaglio ha evidenziato un picco specifico ad una temperatura di Melting $\left(\mathrm{T}_{\mathrm{m}}\right)$ di $81^{\circ} \mathrm{C}$. In tutti gli esperimenti la quantificazione del prodotto specifico è stata eseguita perciò a $78^{\circ} \mathrm{C}$ per evitare la possibile interferenza data dalla sporadica presenza di dimer primer. L'amplificazione, l'acquisizione dei dati e l'analisi sono state effettuate mediante LightCycler della ditta Roche (Mannheim, Germany), con software dedicato (LightCycler 5.3.2 software; Roche). Questo software è in grado di rivelare il threshold cycle $\left(\mathrm{C}_{\mathrm{t}}\right)$, ossia il numero del ciclo dove l'intensità di fluorescenza raggiunge un valore significativo rispetto alla fluorescenza di base. $\mathrm{Il}_{\mathrm{t}}$ è direttamente proporzionale al $\log _{10}$ del numero di copie di partenza. I risultati quantitativi dei campioni sono stati normalizzati come copie di HIV1 DNA/1000000 di cellule.

\section{Analisi statistica}

Per lo studio statistico abbiamo utilizzato il test di Mann-Whitney e l'analisi di correlazione per determinare la relazione tra RNA e DNA.

\section{RISULTATI}

Analisi longitudinale del carico virale plasmatico Tutti i soggetti, inclusi nello studio, già dopo un mese dall'interruzione della terapia presentavano un aumento significativo del carico virale (MannWhitney test $\mathrm{p}=0.036)$ con una media di $4.2 \log _{10}$ rispetto ai $3.5 \log _{10}$ osservati al baseline (tempo $0)$. Alla fine del periodo di osservazione, si è osservato in tutti i soggetti in esame una aumento significativo della viremia plasmatici (figura I).

Analisi longitudinale del carico provirale plasmatico

Contemporaneamente abbiamo quantificato il DNA provirale di tali pazienti, mediante real time PCR, ogni mese dopo l'interruzione della terapia e successivamente ad intervalli costanti di 4 setti- 
mane. I risultati ottenuti hanno mostrato un andamento variabile del DNA viral-load. Due, dei dodici pazienti inclusi nello studio, (tabella 1, soggetti 1 e 10) hanno evidenziato un aumento significativo dei livelli di DNA, sin dal mese successivo all'interruzione del trattamento e fino al termine del periodo di osservazione.

Anche in altri otto pazienti abbiamo potuto osservare un aumento del carico provirale. In questo caso, però a differenza dei soggetti 1 e 10, l'andamento del DNA provirale è risultato fluttuante: in particolare dopo una apparente diminuzione abbiamo osservato, a distanza di 12-20 settimane, un incremento del numero di cellule positive per la presenza di DNA virale (tabella 2: pazienti 2, 3, $5,6,8,9,11$ e 12). Infine in altri due pazienti (tabella 3 , pazienti 4 e 7 ) si è rilevata una diminuzione significativa del carico provirale rispettivamente dopo 20 e 24 settimane.

Non è stata riscontrata alcuna correlazione significativa tra il carico virale e quello provirale (Mann-Whitney test).

\section{Livello dei CD4:}

Tutti i pazienti al momento dell'interruzione del trattamento farmacologico, presentavano valori di CD4 superiori a 400 cellule/mmc. In seguito alla sospensione della terapia si è evidenziata, in tutti, una diminuzione del numero dei CD4. Alla fine del follow-up, le percentuali di riduzione dei CD4 erano le seguenti: $34 \%$ per i campioni 1 e $3,31 \%$ per il $2,39 \%$ per il $4,42 \%$ per il $9,53 \%$ per il 10 . Un moderato declino si è registrato nei campioni $5,6,7$ e 8,11 e 12 con una percentuale variabile dal 18 al 26\%. Nessuna correlazione è stata osservata tra livelli di CD4 e la carica provirale a $D N A$ $(\mathrm{r}=0.5, \mathrm{p}>0.005)$, mentre, come atteso, si è registrata una correlazione inversa, statisticamente significativa, tra il numero di copie di RNA nel plasma ed il numero dei CD4.

\section{DISCUSSIONE}

Il ruolo delle interruzioni terapeutiche nella gestione dell'infezione da HIV è ancora oggetto di molte riflessioni ed analisi. Negli ultimi anni infatti, diversi trial sono stati effettuati al fine di valutare l'efficacia e la sicurezza di programmi di terapia intermittente in pazienti con infezione cronica. In particolare, il bilancio tra $\mathrm{i}$ potenziali benefici ed i possibili rischi di progressione della malattia non è ancora ben definibile. I dati fino ad oggi raccolti mostrano che solo il 10-20\% dei pazienti, che aderiscono a tali protocolli, ottengono una soppressione della replicazione virale $(12,13,17)$.

L'obiettivo del nostro studio è stato quello di seguire un particolare gruppo di pazienti che, spontaneamente, avevano deciso di interrompere la terapia per un periodo variabile dai 4 ai 6 mesi, nonostante presentassero alti livelli di carica virale plasmatica.

In particolar modo, la nostra attenzione si è focalizzata sull'analisi longitudinale del DNA provirale che rappresenta un parametro di notevole importanza per valutare l'andamento dell'infezione e per monitorare la progressione della malattia $(8,7,15,19)$. Infatti, nonostante la determinazione nel plasma dell'RNA virale rappresenti una misura in grado di indicare l'attiva replicazione del virus nei soggetti infetti, la formazione, la stabilità ed il turnover del pool provirale rivestono un ruolo essenziale nella patogenesi da $\operatorname{HIV}(7,21)$. I risultati ottenuti durante il follow-up hanno evidenziato, in tutti i pazienti, un rebound virale, confermato da un aumento della carica virale, presente fin dal primo mese dopo l'interruzione della terapia e protrattosi durante tutto il periodo di osservazione.

D'altra parte, l'analisi sul carico provirale ha mostrato un andamento eterogeneo nei dodici pazienti analizzati. Infatti in due pazienti, in accordo con i dati di letteratura $(8,10,15)$ che sottolineano la possibilità che il fallimento terapeutico si accompagna anche ad un incremento del DNA provirale, si è assistito ad un evidente aumento del numero di copie di DNA rispetto al valore di baseline.

In altri otto pazienti dopo un transitorio calo del numero di copie di DNA, il rebound dei livelli di DNA, che si verifica nei mesi successivi, suggerisce che la terapia può essere in grado di controllare il DNA provirale solo per un limitato periodo di tempo.

Infine, la diminuzione del DNA provirale, nonostante alti valori di RNA in due pazienti, conferma la possibilità, anche verificata da altri autori $(2,8,11$, 18), che il calo del DNA cellulare possa essere riferito alla forma integrata in grado, in assenza di terapia, di replicare portando ad un aumento della viremia plasmatici. Rimane comunque da sottolineare la possibilità che anche in questi soggetti si possa verificare nel tempo un aumento del livello del provirus come quello evidenziato negli 8 soggetti, caratterizzati da un andamento altalenate di DNA.

Pertanto anche se i nostri dati sono limitati ad un gruppo alquanto ristretto di pazienti, la possibilità offerta dalla determinazione quantitativa del DNA provirale non deve essere sottovalutata. Individuare 1'andamento nel tempo e stabilire un eventuale valore soglia di DNA come marker prognostico nella pratica clinica $(19,20)$ porterà indubbiamente importanti informazioni, non solo durante il monitoraggio di pazienti sotto terapia, ma anche in individui inclusi in un protocollo di interruzione della terapia (13).

Alla luce di quanto esposto, l'importanza del 
ruolo svolto dai reservoir cellulari nel mantenimento e nella progressione della malattia da HIV suggerisce la necessità di nuovi approcci terapeutici, in grado di consentire un controllo a lungo termine dell'infezione da HIV e solo una lettura attenta dei diversi parametri virologici, immunologici e clinici potrebbe rappresentare l'arma migliore se non per sconfiggere il virus, sicuramente per contenerne, il più possibile, i danni.

Tabella I. Analisi longitudinale del viral load HIV RNA e DNA, dei livelli di CD4 nei pazienti $N^{\circ}$ I e 10 dal momento della sospensione della terapia (tempo 0) fino alla fine del follow-up.

$\begin{array}{ccc}\begin{array}{c}\text { RNA Viral Load } \\ \text { (copie/ml) }\end{array} & \begin{array}{c}\text { DNA Viral } \\ \text { Load }\end{array} & \begin{array}{c}\mathrm{N}^{\circ} \text { dei CD4 } \\ \left(\times 10^{6} \mathrm{cells} / \mathrm{L}\right)\end{array} \\ & \text { (copie/I06PMBCs) } & \end{array}$

Pt I

\begin{tabular}{llll}
\hline tempo0 & 6300 & & 335 \\
\hline tempol & 20800 & 1600 & 324 \\
\hline tempo2 & 30000 & 2200 & 324 \\
\hline tempo3 & 58000 & 1800 & 340 \\
\hline tempo4 & 100000 & 2000 & 300 \\
\hline tempo5 & 120000 & 3800 & 220 \\
\hline $\mathbf{P t I 0}$ & & & \\
\hline tempo0 & 1130 & & 578 \\
\hline tempol & 69000 & 612 & 420 \\
\hline tempo2 & 66000 & 1200 & 320 \\
\hline tempo3 & 100000 & 1600 & 350 \\
\hline tempo4 & 210000 & 2800 & 267 \\
\hline
\end{tabular}

Tabella 3. Analisi longitudinale del carico virale e provirale HIV RNA e DNA, dei livelli di CD4 nei pazienti ( $N^{\circ} 4$ e 7) dal momento della sospensione della terapia (tempo 0) fino alla fine del follow-up.

\begin{tabular}{lccc}
\hline & $\begin{array}{c}\text { HIV-I RNA } \\
\text { (copie/ml) }\end{array}$ & $\begin{array}{c}\text { HIV-I DNA } \\
\text { (copie/I0'PMBCs) }\end{array}$ & $\begin{array}{c}\mathrm{N}^{\circ} \text { dei CD4 } \\
\left(\times 10^{\circ} \text { cells/L) }\right.\end{array}$ \\
\hline $\mathbf{P t 4}$ & & & 572 \\
\hline tempo0 & 1220 & 1300 & 570 \\
\hline tempol & 1400 & 1890 & 522 \\
\hline tempo2 & 24000 & 960 & 572 \\
\hline tempo3 & 46000 & 500 & 576 \\
\hline tempo4 & 27000 & 700 & 432 \\
\hline tempo5 & 32000 & 550 & 348 \\
\hline tempo6 & 31000 & & \\
\hline Pt 7 & & & 462 \\
\hline tempo0 & 1600 & 920 & 450 \\
\hline tempol & 6800 & 750 & 456 \\
\hline tempo2 & 1700 & 910 & 384 \\
\hline tempo3 & 4400 & 690 & 380 \\
\hline tempo4 & 4000 & 400 & 399 \\
\hline tempo5 & 2700 & 300 & 342 \\
\hline tempo6 & 2800 & &
\end{tabular}

Tabella 2. Analisi longitudinale del viral load HIV RNA e DNA, dei livelli di CD4 nei pazienti $N^{\circ} 2,3,5,6,8,9,1 /$ e 12 dal momento della sospensione della terapia (tempo 0 ) fino alla fine del follow-up.

$\begin{array}{ccc}\begin{array}{c}\text { RNA Viral Load } \\ (\text { copie/ml })\end{array} & \begin{array}{c}\text { DNA Viral } \\ \text { Load } \\ \text { (copie/l06PMBCs })\end{array} & \begin{array}{c}\mathrm{N}^{\circ} \text { dei CD4 } \\ \left(\times 10^{6} \text { cells/L) }\right.\end{array}\end{array}$

Pt2

\begin{tabular}{llll}
\hline tempo0 & 11000 & & 345 \\
\hline tempol & 22000 & 1200 & 345 \\
\hline tempo2 & 70000 & 1900 & 365 \\
\hline tempo3 & 100000 & 1500 & 256 \\
\hline tempo4 & 150000 & 1000 & 304 \\
\hline tempo5 & 148000 & 770 & 240 \\
\hline tempo6 & 170000 & 990 & 238 \\
\hline $\mathbf{P t 3}$ & & &
\end{tabular}

tempo0 3600

\begin{tabular}{lccc}
\hline tempo0 & 3600 & & 551 \\
\hline tempol & 43000 & 2300 & 448 \\
\hline tempo2 & 58100 & 820 & 266 \\
\hline tempo3 & 49000 & 580 & 560
\end{tabular}

\begin{tabular}{llll}
\hline tempo3 & 49000 & 580 & 560 \\
\hline tempo4 & 58000 & 990 & 360 \\
\hline
\end{tabular}

Pt5

\begin{tabular}{lccc}
\hline tempo0 & 4900 & & 494 \\
\hline tempol & 93000 & 1500 & 528 \\
\hline tempo2 & 100000 & 1050 & 483 \\
\hline tempo3 & 150000 & 830 & 460 \\
\hline tempo4 & 250000 & 1000 & 404
\end{tabular}

Pt6

\begin{tabular}{lccc}
\hline tempo0 & 2940 & & 625 \\
\hline tempol & 2500 & 1100 & 625 \\
\hline tempo2 & 16000 & 1400 & 529 \\
\hline tempo3 & 7800 & 630 & 625 \\
\hline tempo4 & 25000 & 620 & 552 \\
\hline tempo5 & 24000 & 730 & 750 \\
\hline tempo6 & 17000 & 890 & 483 \\
\hline
\end{tabular}

Pt8

\begin{tabular}{lccc}
\hline tempo0 & 3970 & & 616 \\
\hline tempol & 4000 & 1000 & 616 \\
\hline tempo2 & 85000 & 660 & 532 \\
\hline tempo3 & 100000 & 490 & 361 \\
\hline tempo4 & 84000 & 230 & 420 \\
\hline tempo5 & 74000 & 330 & 456 \\
\hline tempo6 & 100000 & 550 & 460 \\
\hline $\mathbf{P t 9}$ & & & \\
\hline tempo0 & 10500 & & 680 \\
\hline tempol & 14000 & 1500 & 624 \\
\hline tempo2 & 16000 & 1000 & 480 \\
\hline tempo3 & 39000 & 450 & 588 \\
\hline tempo4 & 33000 & 1100 & 520 \\
\hline tempo5 & 145000 & 3100 & 390 \\
\hline $\mathbf{P t I I}$ & & & \\
\hline tempo0 & 10000 & & 480 \\
\hline tempol & 18000 & 1000 & 424 \\
\hline tempo2 & 40000 & 920 & 480 \\
\hline tempo3 & 39000 & 450 & 388 \\
\hline tempo4 & 45000 & 900 & 420 \\
\hline tempo5 & 132000 & 2100 & 370 \\
\hline $\mathbf{P t I 2}$ & & & 640 \\
\hline tempo0 & 10500 & & 624 \\
\hline tempol & 14000 & 1500 & 580 \\
\hline tempo2 & 16000 & 820 & 470 \\
\hline tempo3 & 39000 & 735 & 1500 \\
\hline tempo4 & 33000 & 2200 & \\
\hline tempo5 & 145000 & & \\
\hline & & & 550 \\
\hline
\end{tabular}




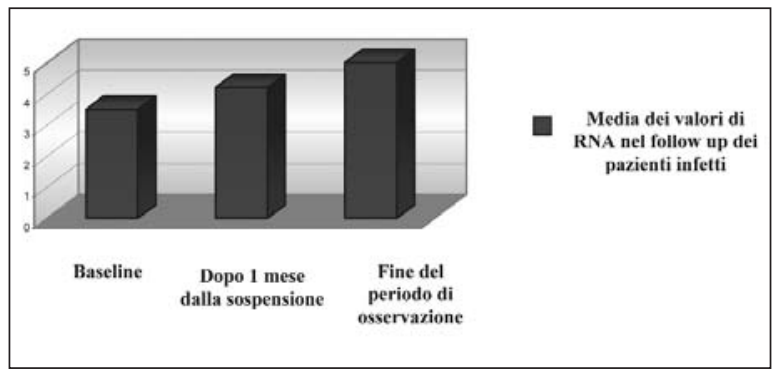

Figura I. Media dei valori di RNA plasmatici al tempo 0 (baseline), dopo I mese dalla sospensione della terapia ed alla fine del follow-up.

\section{BIBLIOGRAFIA}

1. Carcelain G, Tubiana R, Samri A, et al. Transient mobilization of human immunodeficiency virus (HIV)-specific CD4 T-helper cells fails to control virus rebounds during intermittent antiretroviral therapy in chronic HIV type 1 infection. J Virol 2001; 75: 234-41.

2. Florence E, Garcia F, Plana M, et al. Long-term clinical follow-up, without antiretroviral therapy, of patients with chronic HIV-1 infection with good virological response to structured treatment interruption. Clin Infect Dis 2004; 39: 569-74.

3. Furtado MR, Callaway DS, Phair JP, et al. Persistence of HIV-1 transcription in peripheral-blood mononuclear cells in patients receiving potent antiretroviral therapy. N Engl J Med 1999; 340: 1614-22.

4. Gallo RC. New concepts for future control of HIV derived from studies of pathogenesis. Int J Antimicrob Agents 2000; 16: 421-3.

5. Gibellini D, Vitone F, Schiavone P, Ponti C, La Placa M, Re MC. Quantitative detection of human immunodeficiency virus type 1 (HIV-1) proviral DNA in peripheral blood mononuclear cells by SYBR green realtime PCR technique. J Clin Virol 2004; 29: 282-9.

6. Gibellini D, Vitone F, Schiavone P, Ponti C, La Placa M, Re MC. Quantitative detection of human immunodeficiency virus type 1 (HIV-1) viral load by SYBR green real-time RT-PCR technique in HIV-1 seropositive patients. J Virol Methods 2004; 115: 183-9.

7. Hatzakis AE, Touloumi G, Pantazis N, et al. Cellular HIV-1 DNA load predicts HIV-RNA rebound and the outcome of highly active antiretroviral therapy. AIDS 2004; 18: 2261-2267.

8. Kabamba-Mukadi B, Henrivaux P, Ruelle J, Delferriere N, Bodeus M, Goubau P. Human immunodeficiency virus type 1 (HIV-1) proviral DNA load in purified CD4+ cells by LightCycler real-time PCR. BMC Infect Dis 2005; 21: 15.

9. Kostrikis LG, Touloumi G, Karanicolas R, et al. Quantitation of human immunodeficiency virus type 1 DNA forms with the second template switch in peripheral blood cells predicts disease progression independently of plasma RNA load. J Virol 2002; 76: 10099-108.

10. McDermott JL, Giri AA, Martini I, et al. Level of human immunodeficiency virus DNA in peripheral blood mononuclear cells correlates with efficacy of antiretroviral therapy. J Clin Microbiol 1999; 37: 2361-5.

11. Nickle DC, Shriner D, Mittler JE, Frenkel LM, Mullins JI. Importance and detection of virus reser- voirs and compartments of HIV infection. Curr Opin Microbiol 2003; 6: 410-6.

12. Ortiz GM, Wellons M, Brancato J. Structured antiretroviral treatment interruptions in chronically HIV-1infected subjects. Proc Natl Acad Sci U S A 2001; 98 : 13288-93.

13. Oxenius A, Hirschel B. Structured treatment interruptions in HIV infection: benefit or disappointment? Expert Rev Anti Infect Ther 2003; 1: 129-39.

14. Palella FJ Jr, Delaney KM, Moorman AC, et al. Declining morbidity and mortality among patients with advanced human immunodeficiency virus infection. HIV Outpatient Study Investigators.N Engl J Med 1998 Mar 26; 338(13): 853-60.

15. Pellegrin I, Caumont A, Garrigue I, et al. Predictive value of provirus load and DNA human immunodeficiency virus genotype for successful abacavir-based simplified therapy. J Infect Dis 2003; 187: 38-46.

16. Ramratnam B, Mittler JE, Zhang, et al. The decay of the latent reservoir of replication-competent HIV-1 is inversely correlated with the extent of residual viral replication during prolonged anti-retroviral therapy. Nat Med 2000; 6: 82-5.

17. Strain MC, Little SJ, Daar ES, et al. Effect of Treatment, during Primary Infection, on Establishment and Clearance of Cellular Reservoirs of HIV-1. J Infect Dis 2005; 191: 1410-8.

18. Teo IA, Morlese J, Choi JW, Shaunak S. Reliable and reproducible LightCycler qPCR for HIV-1 DNA 2-LTR circles. J Immunol Methods 2002; 1; 270: 109-18.

19. Tierney C, Lathey JL, Christopherson C, et al. Prognostic value of baseline human immunodeficiency virus type 1 DNA measurement for disease progression in patients receiving nucleoside therapy. $\mathrm{J}$ Infect Dis 2003; 187: 144-8.

20. Vitone F, Gibellini D, Schiavone P, Re MC. Quantitative DNA proviral detection in HIV-1 patients treated with antiretroviral therapy. J Clin Virol 2005; 33: 194-200.

21. Wong JK, Hezareh M, Gunthard HF, et al. Recovery of replication-competent HIV despite prolonged suppression of plasma viremia. Science 1997; 278: 1291-5.

\section{Francesca Vitone}

Sezione di Microbiologia Dipartimento di Medicina Clinica Specialistica e Sperimentale Via Massarenti 9, 40138 Bologna Tel. 051 4290900; Fax 051307397 E-mail: vitonfra@yahoo.it 